Pacific Journal of Mathematics

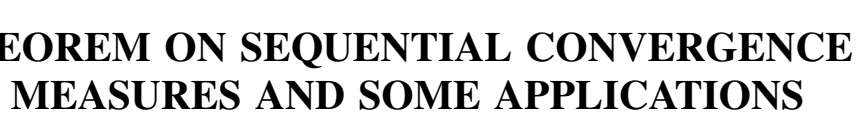




\title{
A THEOREM ON SEQUENTIAL CONVERGENCE OF MEASURES AND SOME APPLICATIONS
}

\author{
JoHN B. CoNwAY
}

If $S$ is a locally compact Hausdorff space, let $\beta S$ be its Stone-Cech compactification and let $M(S)$ be the space of all finite complex valued regular Borel measures on $S$. In this paper we will prove that whenever $S$ is paracompact and $\left\{\mu_{n}\right\}$ is a sequence in $M(\beta S)$ which converges to zero in the weak star topology, then $\lim \int_{S} f d \mu_{n}=0$ for every continuous function $f$, and $\left\{\mu_{n}\right\}$ satisfies a certain uniformity condition on $S$. This generalizes a result of $R$. S. Phillips on weak star sequential convergence in the dual of $l^{\infty}$. Moreover, by using our theorem we can obtain many previously known results whose proofs, though all similar, were apparently independent.

Indeed, the motivation for undertaking the research which led to this paper came from the similarity of the proofs of a number of theorems. Among these is the following.

Theorem A. If $S$ is paracompact and $H$ is a subset of $M(S)$ which is countably compact in the $C(S)$ topology on $M(S)$ then for every $\varepsilon>0$ there is a compact set $K_{\varepsilon} \subset S$ such that

$$
|\mu|\left(S-K_{\varepsilon}\right)<\varepsilon
$$

for every $\mu$ in $H$.

This theorem was first proved by LeCam [15] and then, independently, by the present author [4]. Another result which utilizes the same method of proof referred to above is

THeOREM B. If $S$ is paracompact and $A$ is a closed linear subspace of $C(\beta S)$ such that $A$ interpolates $\beta S-S$ then there is a closed neighborhood $V$ of $\beta S-S$ such that $A$ interpolates $V$.

Note. To say that a closed linear subspace $A$ of $C(\beta S)$ interpolates a closed set $K \subset \beta S$ means that any $f$ in $C(K)$ has an extension $F$ to $\beta S$ such that $F$ is in $A$.

Theorem $B$ is due to Bade [1] for $\sigma$-compact spaces. In addition to these two results there are still others which fit into this same category. These will be presented in $\S 3$ together with the proofs using the main theorem of this paper. However, we have not yet been able to discover a proof of Theorem A based on this result. 
Theorem B can be proved by means of Theorem 2.2, but we have postponed this to a later paper.

1. Preliminaries. The original proofs of the two theorems above, as well as those of some of the theorems in $\S 3$, employ the following approach: Using some previous result; an embedding of $l^{\infty}$ into $C(S)$, the space of bounded continuous complex valued functions on $S$, is constructed. The adjoint of this map is examined and a result of R. S. Phillips is applied to achieve the desired conclusion. This result of Phillips is the following (see [5, p. 32] for a proof).

THEOREM 1.1. If $\left\{\mu_{n}\right\}$ is a sequence in $M(\beta N)$ which converges to zero in the weak * topology of $M(\beta N)$ then

$$
\lim _{m \rightarrow \infty} \sum_{k=m}\left|\mu_{n}(\{k\})\right|=0
$$

uniformly in $n$.

Here we have taken liberty to identify $l^{\infty}$ with $C(N)$ and $\left(l^{\infty}\right)^{*}$ with $M(\beta N) ; N$ is the set of positive integers with the discrete topology. The proof of our theorem will also use this result.

The notation and terminology used here will be standard as is found in [9], [12], and [14]; however, there will be a few minor deviations. We will denote by $C_{0}(S)$ those functions in $C(S)$ which vanish at infinity, and $C_{00}(S)$ will be the space of continuous functions having compact support. The term "weak * topology" will be restricted to mean only the $\sigma(M(\beta S), C(\beta S))$ topology on $M(\beta S)$. The $\sigma\left(M(S), C_{0}(S)\right)$ and $\sigma(M(S), C(S))$ topologies on $M(S)$ will be referred to as the $C_{0}$-weak $*$ and $C$-weak $*$ topologies respectively. Notice that if we consider $M(S)$ as a subset of $M(\beta S)$ then the $C$ weak $*$ topology is exactly the relativisation of the weak $*$ topology to $M(S)$.

If $\mu$ is in $M(S)$ and $B$ is a Borel subset of $S$ then $\mu_{B}$ denotes the restriction of $\mu$ to $B$. That is, $\mu_{B}(A)=\mu(B \cap A)$ for every Borel set $A$. To simplify our notation, we will not use any symbol to distinguish between a function in $C(S)$ and its extension to $\beta S$.

Finally, we wish to state the characterization of paracompact locally compact spaces needed below. The proof may be found in [9, p. 241].

THEOREM 1.2. A locally compact space $S$ is paracompact if and only if $S=\bigcup_{\alpha} S_{\alpha}$ where the $S_{\alpha}$ are pairwise disjoint open and closed $\sigma$-compact subsets of $S$. 
2. The main theorem. If $H$ is a subset of $M(S)$ then we say that $H$ is tight if and only if $H$ is uniformly bounded and for every $\varepsilon>0$ there is a compact set $K_{\varepsilon} \subset S$ such that $|\mu|\left(S-K_{\varepsilon}\right)<\varepsilon$ for every $\mu$ in $H$. (In [4] the author showed a relationship between tightness and the strict topology on $C(S))$. With this definition we state a well-known lemma whose proof will not be given.

LEMMA 2.1. Let $\left\{\mu_{n}\right\}$ be a sequence in $M(S)$ which is tight and let $\mu$ be in $M(S)$ such that

$$
\lim _{n \rightarrow \infty} \int_{S} \varphi d \mu_{n}=\int_{S} \phi d \mu
$$

for each $\phi$ in $C_{00}(S)$. Then $\left\{\mu_{n}\right\}$ converges to $\mu C$-weak *.

We now give the main result.

THEOREM 2.2. If $S$ is paracompact and $\left\{\mu_{n}\right\}$ is a sequence in $M(\beta S)$ which converges weak $*$ to $\mu$ in $M(\beta S)$ then $\left\{\mu_{n_{S}}\right\}$ is tight and converges $C$-weak $*$ to $\mu_{s}$.

REMARKS. It is easily seen that $\lim \int_{S} \varphi d \mu_{n}=\int_{S} \varphi d \mu$ for each $\varphi$ in $C_{00}(S)$. Hence, by Lemma 2.1, the important part of the result is that $\left\{\mu_{n_{S}}\right\}$ is tight. A moments reflection will show that tightness in $M(N)=l^{1}$ is exactly the uniform limit condition in Phillips' theorem. Therefore this is a generalization of Theorem 1.1.

Proof. As was noted in the above remarks we need only show that $\left\{\mu_{n_{S}}\right\}$ is tight. Since this proof is similar to that of another result of the author [4, p. 478], we will omit many of the details. As in [4] we will only consider the case where $S$ is $\sigma$-compact, the general theorem being accomplished by means of Theorem 1.2. Therefore, let $S=\bigcup_{n=1} D_{n}$ where each $D_{n}$ is compact and $D_{n}$ is contained in int $D_{n+1}$ (the interior of $D_{n+1}$ ).

Suppose $\left\{\mu_{n_{S}}\right\}$ is not tight; since $\left\{\mu_{n}\right\}$ is uniformly bounded there is an $\varepsilon>0$ such that for every $k \geqq 1$ there is a $\mu_{n_{k}}$, subsets $K_{k}$ and $U_{k}$ of $S$, and a function $\varphi_{k}$ having the properties: empty;

(a) $K_{k}$ is compact, $U_{k}$ is open in $S$ with $U_{-\bar{c}}$ compact and $U_{\bar{k}}^{-} \cap K_{k}$

(b) $D_{k} \cup K_{k} \cup U_{k}^{-} \subset \operatorname{int} K_{k+1}$;

(c) $\left|\mu_{n_{k}}\left(U_{k}\right)\right|>\varepsilon / 4$;

(d) $\varphi_{k}$ is in $C_{00}(S),\left\|\varphi_{k}\right\|_{\infty}=1$, the support of $\varphi_{k}$ is contained in $U_{k}$ and $\left|\mu_{n_{k}}\right|\left(U_{k}\right)<\left|\int_{S} \phi_{k} d \mu_{n_{k}}\right|+\varepsilon / 8$. If for every $\xi=\left\{x_{k}\right\}$ in 
$l^{\infty}$ and $s \in S$ we define

$$
T(\xi)(s)=\sum_{k=1}^{\infty} x_{k} \varphi_{k}(s)
$$

then $T(\xi)$ is in $C(S)$ and $\|T(\xi)\|_{\infty}=\|\xi\|_{\infty}$. It follows that $T$ is an isometry of $l^{\infty}$ into $C(S)$. Therefore $T^{*}: M(\beta S) \rightarrow\left(l^{\infty}\right)^{*}=M(\beta N)$ exists and is weak $*$ continuous. From our hypothesis on $\left\{\mu_{n}\right\}$ it follows that $\left\{T^{*}\left(\mu_{n_{k}}\right)\right\}$ converges to $T^{*}(\mu)$ weak $*$ in $M(\beta N)$. It is an easy matter to check that

$$
T^{*}\left(\mu_{n_{k}}\right)(\{j\})=\int_{\beta S} \Phi_{j} d \mu_{n_{k}}=\int_{S} \varphi_{j} d \mu_{n_{k}} \cdot
$$

Applying Theorem 1.1 we get that

$$
\lim _{m \rightarrow \infty} \sum_{j=m}^{\infty}\left|\int_{S} \varphi_{j} d \mu_{n_{k}}\right|=0
$$

uniformly in $k$. In particular, we have that

$$
\lim _{k \rightarrow \infty} \int_{S} \varphi_{k} d \mu_{n_{k}}=0
$$

which, when combined with condition (d) above, yields a contradiction to (c). This completes the proof of the theorem.

3. Applications. The applications here are to sequential convergence in $M(S)$. For further results and references concerning this topic the reader is referred to the papers of Varadarajan [16] and Dudley [8].

The first result is due to Diendonné [7]. It should be noted that his original proof was a generalization of a proof of a theorem of Schür. However, it is an immediate consequence of Theorem 2.2.

TheOREM 3.1. If $S$ is paracompact and $\left\{\mu_{n}\right\}$ is a C-weak * convergent sequence in $M(S)$ then $\left\{\mu_{n}\right\}$ is tight.

The next theorem was proved independently by Varadarajan [16, p. 195] and Collins and Dorroh [2]. Both have different proofs; Varadarajan uses a method of LeCam [15, p. 218] and Collins and Dorroh use the method of Theorem 2.2.

THEOREM 3.2. If $S$ is paracompact then $M(S)$ is C-weak * sequentially complete. Moreover, if $\left\{\mu_{n}\right\}$ is a C-weak * Cauchy sequence then $\left\{\mu_{n}\right\}$ is tight.

Proof. If $\left\{\mu_{n}\right\}$ is a $C$-weak $*$ Cauchy sequence in $M(S)$ then 
consider each $\mu_{n}$ as an element in $M(\beta S)$. Since $\sup \left\{\left\|\mu_{n}\right\|: n \geqq 1\right\}<\infty$, Alagolu's theorem implies that there is a $\mu$ in $M(\beta S)$ which is a weak * cluster point of $\left\{\mu_{n}\right\}$. But for each $f$ in $C(S)$,

$$
\lim _{n \rightarrow \infty} \int_{\beta S} f d \mu_{n}=\lim _{n \rightarrow \infty} \int_{S} f d \mu_{n}
$$

exists and so $\left\{\mu_{n}\right\}$ converges to $\mu$ weak $*$ in $M(\beta S)$. Therefore, Theorem 2.2 implies that $\left\{\mu_{n}\right\}$ converges to $\mu_{S} C$-weak $*$ in $M(S)$ and $\left\{\mu_{n}\right\}$ is tight. This completes the proof.

The following theorem of Grothendieck [11] characterizes the weakly convergent sequences in $M(S)$ (i.e., the sequences which converge for the $\sigma\left(M(S), M(S)^{*}\right)$ topology).

THeOREM 3.3. If $S$ is an arbitrary locally compact space and $\mu, \mu_{1}, \mu_{2}, \cdots$ are elements in $M(S)$, then the following are mutually equivalent:

(a) $\left\{\mu_{n}\right\}$ converges to $\mu$ weakly in $M(S)$;

(b) $\left\{\mu_{n}\right\}$ is uniformly bounded and $\mu(U)=\lim \mu_{n}(U)$ for every open $F_{\sigma}$ set $U \subset S$; function $f$;

(c) $\lim \int_{S} f d \mu_{n}=\int_{S} f d \mu$ for every bounded lower semicontinuous

(d) (i) $\left\{\mu_{n}\right\}$ is tight, (ii) $\left\{\mu_{n}\right\}$ converges to $\mu C$-weak $*$, and (iii) for every $\varepsilon>0$ and every compact set $K \subset S$ there is an open set $V_{\varepsilon} \supset K$ such that $\left|\mu_{n}\right|\left(V_{\varepsilon}-K\right)<\varepsilon$ for every $n \geqq 1$.

Proof. It is trivial to see that (a) implies (b) and that (b) and (c) are equivalent. Therefore, assume that (c) holds and let us prove (d). Since we are only dealing with a countable family of regular measures we can find an open $\sigma$-compact set $S_{1} \subset S$ such that

$$
|\mu|\left(S-S_{1}\right)=\left|\mu_{n}\right|\left(S-S_{1}\right)=0
$$

for every $n \geqq 1$. If $f$ is a nonnegative function of $S$ which is continuous on $S_{1}$ and vanishes on $S-S_{1}$, then $f$ is lower semicontinuous. From this we have that $\left\{\mu_{n}\right\}$ converges to $\mu C$-weak $*$ in $M\left(S_{1}\right)$. Since $S_{1}$ is $\sigma$-compact, Theorem 2.2 implies $\left\{\mu_{n}\right\}$ is tight in $M\left(S_{1}\right)$ and hence in $M(S)$.

Since (ii) is clearly true it remains only to prove (iii). By means of (i), the proof of the general case requires only minor technical changes in the proof of the case when $S$ is compact. Hence, let us make this assumption. Let $\varepsilon>0$ and let $K$ be a given compact subset of $S$. By passing to a larger compact set with the same measure as $K$, we may assume that $K$ is a $G_{j}$ set. But then $W=S-K$ is an 
open $\sigma$-compact subset of $S$. As above, it follows that $\left\{\mu_{n_{W}}\right\}$ converges to $\mu_{W}$ C-weak $*$ in $M(W)$. Hence $\left\{\mu_{n_{W}}\right\}$ is tight in $M(W)$; that is, there is a compact set $D \subset W$ such that $\left|\mu_{n}\right|(W-D)<\varepsilon$ for every $n$. But if $V=S-D, V$ is open, $K \subset V$ and $\left|\mu_{n}\right|(V-K)=$ $\left|\mu_{n}\right|(W-D)<\varepsilon$ for each $n$.

Now let us assume that (d) is true and prove (a). As before we will only consider the case where $S$ is compact; we will further assume that $\mu=0$. Observe that with $S$ compact condition (iii) is equivalent to the following:

(iii)' for every $\varepsilon>0$ and every open set $U$ there is a compact set $K \subset U$ such that $\left|\mu_{n}\right|(U-K)<\varepsilon$ for each $n$.

Using (iii)', Urysohn's Lemma, and property (ii) we get that $\lim \mu_{n}(U)=0$ for every open set $U$. It remains to show that this implies that $\lim \mu_{n}(A)=0$ for each Borel set $A$ in $S$ (see [10, p. 308]). But again we need only show this when $A=\cup K_{n}$, where each $K_{n}$ is compact and $K_{n} \subset K_{n+1}$. Let $\varepsilon>0$ and for each $n$ choose an open set $V_{n} \supset K_{n}$ such that for every $k \geqq 1$

$$
\left|\mu_{k}\right|\left(V_{n}-K_{n}\right)<\varepsilon\left(\frac{1}{2}\right)^{n+1} \text {. }
$$

Let $V=\bigcup_{n=1}^{\infty} V_{n}$; then $V$ is open, $A \subset V$ and for every $k$

$$
\begin{aligned}
\left|\mu_{k}\right|\left(V_{n}-A\right) & =\left|\mu_{k}\right|\left(\bigcap_{j=1}^{\infty}\left(V_{n}-K_{j}\right)\right) \\
& \leqq\left|\mu_{k}\right|\left(V_{n}-K_{n}\right) \\
& \leqq \varepsilon\left(\frac{1}{2}\right)^{n+1} .
\end{aligned}
$$

Thus,

$$
\left|\mu_{k}\right|(V-A)=\left|\mu_{k}\right|\left(\bigcup_{n=1}^{\infty}\left(V_{n}-A\right)\right) \leqq \frac{1}{2} \varepsilon
$$

for all k. But $\lim \mu_{n}(V)=0$ and so $\left|\mu_{n}(A)\right|<\varepsilon$ for sufficiently large $n$. This completes the proof of the theorem.

REMARKs. The proof that (d) implies (a) does not employ Theorem 2.2, but was given here for completeness. Dieudonné [6] showed that (c) and (d) are equivalent for $S$ compact and that (a) and (b) are equivalent when $S$ is a compact metric space. In fact, when $S$ is metrisable he showed that the condition of uniform boundedness in (b) can be dropped.

A compact space $S$ is said to be a $\sigma$-Stonian space if and only 
if every bounded sequence of continuous real valued functions has a supremum. This is equivalent to the proposition that the closure of every open $F_{\sigma}$ set is open. One interesting property of a $\sigma$-Stonian space is that if $U$ is an open $F_{\sigma}$-set then $\beta U$ is exactly $U^{-}$, the closure of $U$. Using this fact and our Theorem 2.2 we can prove the following result of Grothendieck [11, p. 168] and Isbell and Semadeni $[13$, p. 46].

THeOREM 3.4. If the compact space $S$ is a $\sigma$-Stonian space and $\left\{\mu_{n}\right\}$ is a sequence in $M(S)$ which converges weak $*$ then $\left\{\mu_{n}\right\}$ converges weakly.

Proof. Let $\mu$ be the weak $*$ limit of $\left\{\mu_{n}\right\}$. By the preceding theorem we must show that $\mu(U)=\lim \mu_{n}(U)$ whenever $U$ is an open $F_{\sigma}$ set. But then $\beta U=U^{-}$and $U^{-}$is open in $S$. Hence, if $\nu_{n}$ (respectively, $\nu$ ) is the restriction of $\mu_{n}$ (respectively, $\mu$ ) to $U^{-}$we have that $\left\{\nu_{n}\right\}$ converges to $\nu$ weak $*$ in $M(\beta U)$. Therefore, by Theorem 2.2 we have that $\left(\nu_{n_{U}}\right\}$ converges to $\nu_{U} C$-weak $*$ in $M(U)$. In particular, we get that $\mu(U)=\lim \mu_{n}(U)$. This completes the proof of the theorem.

REMARKs. Grothendieck proved this for $S$ a Stonian space and Isbell and Semandeni showed it to be true in the more general $\sigma$ Stonian spaces.

As a final application we give a generalization of Phillips' theorem that $c_{0}$ is not complemented in $l^{\infty}$.

THEOREM 3.5. If $S$ is paracompact and not compact then there is no bounded projection from $C(S)=C(\beta S)$ onto $C_{0}(S)$.

Proof. Suppose $P$ is a bounded projection from $C(\beta S)$ onto $C_{0}(S)$. Then $P^{*}: M(S) \rightarrow M(\beta S)$ is a bounded linear map which is $C_{0}$-weak *weak $*$ continuous. Also, if $\phi$ is in $C_{0}(S)$ and $u$ is in $M(S)$ we have

$$
\int_{\beta S} \varphi d P^{*}(\mu)=\int_{S} \varphi d \mu \text {. }
$$

Therefore $P^{*}(\mu)_{S}=\mu$ for all $\mu$ in $M(S)$. But since $S$ is paracompact and not compact we can, by Theorem 1.2 , choose a sequence $\left\{s_{n}\right\}$ in $S$ which is eventually in the complement of every compact subset of $S$. Hence, $\lim \varphi\left(s_{n}\right)=0$ for every $\varphi$ in $C_{0}(S)$. If $\mu_{n}$ is the unit point mass at $s_{n}$ then $\left\{\mu_{n}\right\}$ converges $C_{0}$-weak $*$ to zero in $M(S)$. Thus, $\left\{P^{*}\left(\mu_{n}\right)\right\}$ converges to zero weak $*$ in $M(\beta S)$. By Theorem 2.2 we have that $\left\{\mu_{n}\right\}=\left\{P^{*}\left(\mu_{n}\right)_{s}\right\}$ converges to zero $C$-weak $*$ in $M(S)$ and is tight. Clearly this is a contradiction and the theorem is proved. 
REMARKS. This proof is the analogue of Phillips' proof that $c_{0}$ is not complemented in $l^{\infty}$, where Theorem 2.2 is used in place of Phillips' Theorem 1.1. In [3] the present author has proved a more general result than Theorem 3.5. Also Theorem 3.5 can be obtained as a corollary to Bade's Theorem B.

\section{BIBLIOGRAPHY}

1. W. G. Bade, Extension of interpolating sets, Functional Analysis-Proceedings of a conference held at the University of California, Irvine; edited by B. R. Gelbaum, Thompson Book Company, Washington, D.C. 1967.

2. H. S. Collins and J. R. Dorroh, Remarks on certain function spaces, Math. Ann. 176 (19768), 87-95.

3. J. B. Conway, Projection and retractions, Proc. Amer. Math. Soc. 17 (1966), 843-847.

4. - The strict topology and compactness in the space of measures II, Trans. Amer. Math. Soc. 126 (1967), 474-486.

5. M. M. Day, Normed linear spaces, Springer-Verlag, Berlin, 1958.

6. J. Dieudonné, Sur la convergence des suites measures de Radon, Anais da Acad. Bras. di Ci. 23 (1951), 21-38 and 277-282.

7. - Sur le produit de composition, Composition Math. 12 (1954), 17-34.

8. R. M. Dudley, Convergence of Baire measures, Studia Math. 27 (1966), 251-268.

9. J. Dugundi, Topology, Allyn and Bacon, Inc., Boston, 1966.

10. N. Dunford and J. Schwartz, Linear operators, Part I. Interscience, New York, 1958.

11. A. Grothendieck, Sur les applications linéaires faiblement compactes d'espaces du type $C(K)$, Canad. J. Math. 5 (1953), 129-173.

12. E. Hewitt and K. Stromberg, Real and abstract analysis, Springer-Verlag, New York, 1965.

13. J. Isbell and Z. Semadeni, Projection constants and spaces of continuous functions, Trans. Amer. Math. Soc. 107 (1963), 38-48.

14. J. L. Kelley, I. Namioka, et al, Linear topological spaces, D. Van Nostrand Co., Inc., Princeton, 1963.

15. L. LeCam, Convergence in distribution of stochastic processes, Univ. of California Publications in Statistics, 2 (1957), 207-236.

16. V. S. Varadarajan, Measures on topological spaces, Amer. Math. Soc. Translations, Series 2, 48 (1965), 161-228.

Received December 11, 1967. This research was done while the author held a Summer Faculty Fellowship from Indiana University

INDIANA UNIVERSITY

BLOOMINGTON, INDIANA 


\section{PACIFIC JOURNAL OF MATHEMATICS}

\section{EDITORS}

H. ROYDEN

Stanford University

Stanford, California

R. R Phelps

University of Washington

Seattle, Washington 98105
J. DUGUNDJI

Department of Mathematics

University of Southern California

Los Angeles, California 90007

RICHARD ARENS

University of California

Los Angeles, California 90024

\section{ASSOCIATE EDITORS}
E. F. BECKENBACH
B. H. NeumanN
F. WOLF
K. YosidA

\section{SUPPORTING INSTITUTIONS}

UNIVERSITY OF BRITISH COLUMBIA

CALIFORNIA INSTITUTE OF TECHNOLOGY

UNIVERSITY OF CALIFORNIA

MONTANA STATE UNIVERSITY

UNIVERSITY OF NEVADA

NEW MEXICO STATE UNIVERSITY

OREGON STATE UNIVERSITY

UNIVERSITY OF OREGON

OSAKA UNIVERSITY

UNIVERSITY OF SOUTHERN CALIFORNIA
STANFORD UNIVERSITY

UNIVERSITY OF TOKYO

UNIVERSITY OF UTAH

WASHINGTON STATE UNIVERSITY

UNIVERSITY OF WASHINGTON

AMERICAN MATHEMATICAL SOCIETY CHEVRON RESEARCH CORPORATION TRW SYSTEMS

NAVAL WEAPONS CENTER 


\section{Pacific Journal of Mathematics

Vol. 28, No. $1 \quad$ March, 1969

Patrick Robert Ahern, On the geometry of the unit ball in the space of real annihilating measures .............................. 1

Kirby Alan Baker, Equational classes of modular lattices ............. 9

E. F. Beckenbach and Gerald Andrew Hutchison, Meromorphic minimal surfaces ......................................... 17

Tae Ho Choe, Intrinsic topologies in a topological lattice ..............

John Bligh Conway, A theorem on sequential convergence of measures and

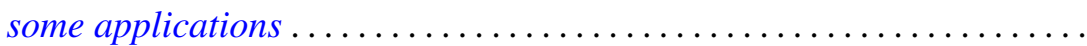

Roger Cuppens, On the decomposition of infinitely divisible probability laws without normal factor.

Lynn Harry Erbe, Nonoscillatory solutions of second order nonlinear

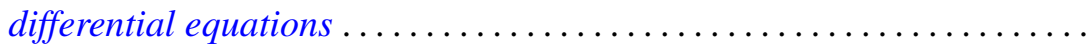

Burton I. Fein, The Schur index for projective representations of finite

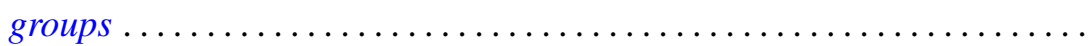

Stanley P. Gudder, A note on proposition observables............... 101

Kenneth Kapp, On Croisot's theory of decompositions ............... 105

Robert P. Kaufman, Gap series and an example to Malliavin's theorem . . . 117

E. J. McShane, Robert Breckenridge Warfield, Jr. and V. M. Warfield,

Invariant extensions of linear functionals, with applications to measures and stochastic processes ................

Marvin Victor Mielke, Rearrangement of spherical modifications ...

Akio Osada, On unicity of capacity functions ..............

Donald Steven Passman, Some 5/2 transitive permutation groups ...

Harold L. Peterson, Jr., Regular and irregular measures on groups and dyadic spaces...

Habib Salehi, On interpolation of $q$-variate stationary stochastic processes...

Michael Samuel Skaff, Vector valued Orlicz spaces generalized

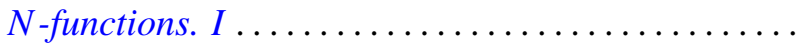

A. J. Ward, On $H$-equivalence of uniformities. II...........

Thomas Paul Whaley, Algebras satisfying the descending chain condition for subalgebras...

G. K. White, On subgroups of fixed index

Martin Michael Zuckerman, A unifying condition for implications among the axioms of choice for finite sets ................. 